\title{
Small-Bowel Obstruction Secondary to Dental Impression Material
}

\author{
Fred Reifsteck, $M D$
}

Abdominal pain is a common complaint at the family physician's office. Small-bowel obstruction is included in the differential diagnosis. Many patients with obstruction have a history of previous abdominal surgeries as the cause of obstruction, but mechanical obstruction is another common cause. Mechanical obstruction can be caused by an ingested foreign body or foodstuff. This case is the first reported of mechanical small-bowel obstruction caused by swallowed dental impression material.

\section{Case Report}

A 63-year-old woman came to her family physician's office complaining of a 4-day history of nonlocalized pressure-like abdominal pain, vomiting, and diarrhea. The diarrhea, which she said was not bloody, had lasted only 1 day and was relieved with bismuth subsalicylate (Pepto-Bismol). She had vomited only two to three times; the vomitus was also not bloody. She was taking only sips of liquids at the time of her initial visit. She believed the symptoms began after eating collard greens, which she thought were spoiled. She denied fever or urinary tract symptoms. She had hypertension that was well controlled with atenolol and lisinopril. She was taking clonazepam for restless legs and conjugated estrogens for hormone replacement.

At examination her weight was within normal range. Her temperature was $98.4^{\circ} \mathrm{F}$, blood pressure $110 / 70 \mathrm{~mm} \mathrm{Hg}$, pulse rate 80 beats per minute, and respirations $18 / \mathrm{min}$. Her mucus membranes were somewhat dry. During an examination of her abdomen, she had hypoactive bowel sounds and mild diffuse tenderness, with some localization to the left lower quadrant, but no rebound, guarding, or

Submitted, revised, 21 April 1999.

From the Section of Family Practice (FR), Southeast Permanente Medical Group, Kaiser Permanente, Jonesboro, Ga. Address reprints request to Fred Reifsteck, MD, Kaiser Permanente, 2400 Mt. Zion Pkwy, Jonesboro, GA 30236. distension. The patient's urinalysis disclosed proteinuria $(1+)$, but there were no white blood cells, and it was negative for nitrites. Her total white blood cell count was $6.3 \times 10^{3} / \mathrm{mL}$ with $84 \%$ granulocytes, her hemoglobin $15.5 \mathrm{~g} / \mathrm{dL}$, and her hematocrit $46.1 \%$. Her abdominal radiograph showed only a nonspecific bowel gas pattern with no air-fluid levels. Her condition was presumptively diagnosed as gastroenteritis. She was treated in the office with intravenous fluids and intravenous promethazine. She began to feel better and was sent home to try clear liquids, continue promethazine suppositories as needed, and return to the office the next day.

She returned to the office the next day feeling worse, stating that she had vomited nine times overnight. She had orthostatic hypotension, with a systolic blood pressure drop of $14 \mathrm{~mm} \mathrm{Hg}$ with position change. A second abdominal radiograph showed air-fluid levels. She was hospitalized for treatment of a small-bowel obstruction.

The patient received intravenous hydration, nasogastric suctioning, and bowel rest, as well as antibiotics for the possibility of diverticulitis. She initially showed improvement on this course. A computed tomographic (CT) scan showed dilated small-bowel loops. She worsened slightly, however, and the distention increased, so a radiographic study of the small intestine using barium was performed. The findings of the study were consistent with small-bowel obstruction at the level of the ileocecal region. After the small-bowel series, she had decreased abdominal distension, felt better, and began to pass barium through her rectum. Shortly thereafter, her symptoms got worse with the conservative treatment, and on day 5 of hospitalization she underwent a laparotomy. During the surgery a foreign body was found at the ileocecal junction. Postoperatively, the patient continued on parenteral nutrition and nasogastric decompression. Her bowel function was somewhat slow to 
resume, but it eventually returned, and a diet of solid food was gradually increased.

The patient later volunteered that 13 days before the onset of the symptoms she had visited her dentist and had five dental impressions made. The dentist supplied the additional information that the impressions were posterior in location and that the impression material was vinyl polysiloxane. The patient stated that she saw one impression block that had material missing when it was removed from her mouth, and she remembers feeling some of the material going down the back of her throat. At the time of the incident, she was unable to tell the dentist. The foreign body removed at the time of surgery was a mass of dental impression-molding material consistent with the vinyl polysiloxane.

\section{Discussion}

A small-bowel obstruction can initially have symptoms and signs similar to those caused by paralytic ileus, acute gastroenteritis, acute appendicitis, or acute pancreatitis. Certain physical findings and diagnostic tests help differentiate these conditions. Crampy abdominal pain, vomiting, obstipation, abdominal distension, and failure to pass flatus are characteristics of small-bowel obstructions. ${ }^{1}$ Proximal small-bowel obstructions tend to cause greater amounts of vomiting and less distension compared with distal obstructions. ${ }^{2}$ Crampy pain followed by explosive diarrhea is usually associated with partial obstruction. Not every case has all these characteristics, as was evident in this case. Radiographs can confirm the diagnosis and better define the site of the obstruction. Patients with bowel obstruction usually have abnormally large quantities of gas in the bowel; however, in mechanical small-bowel obstructions there is minimal to no gas in the colon.

A CT scan can be helpful in diagnosing and determining the location of complete small-bowel obstruction. ${ }^{1}$ The fundamentals of treatment consist of fluid and electrolyte therapy, nasogastric decompression, and timely surgical intervention, if necessary. Only $12 \%$ to $20 \%$ of patients with partial small-bowel obstructions require surgery, with most patients improving within 48 hours. ${ }^{2}$ The timing of surgical intervention depends on three main factors: duration of obstruction and severity of fluid, electrolyte, and acid-base abnormalities; the opportunity to improve vital organ function; and the risk of bowel strangulation. ${ }^{1}$
No other case of intestinal obstruction secondary to dental impression material could be found in a search of the MEDLINE literature. The three most common causes of small-bowel obstruction are postoperative adhesions, hernias, and malignant tumors. Intestinal obstruction can be caused by foodstuff, with an incidence up to $4 \% .^{3}$ Intestinal obstructions can also be caused by solid objects, such as coins and toys, which are most commonly found in children aged 1 to 3 years. ${ }^{3}$ In descending order, the most common locations of obstruction, regardless of the source, are the ileocecal region (where this patient's obstruction occurred), the proximal ileum, and the distal jejunum. The obstructing agent must have a diameter of $2.5 \mathrm{~cm}$ or greater. $^{4}$

A report from England describes an appendicitis caused by a class II amalgam restoration. ${ }^{5}$ It was speculated that the amalgam lodged in a "wide mouthed" appendix. Most dental work or restorations that are swallowed pass directly through the gastrointestinal tract. Vinyl polysiloxane, which is hygroscopic, is the viscous part of a two-component mixture, the other being a thicker plastic-like material. A chemical reaction takes place when the two are combined, making the solid block. It can be postulated that the material in this case became increasingly solid with time, thus making it more difficult for it to pass through the gastrointestinal tract. Because of the solid nature and composition of the vinyl polysiloxane, digestive juices were unable to degrade the material.

In conclusion, care should be taken when using dental impression material, especially in posterior impressions. Blocks should be examined for missing material. If it is suspected that a patient has swallowed some of the material, the patient should be warned to be aware of the symptoms and signs of bowel obstruction. Dental impression material that will not solidify in the gastrointestinal tract and that can be degraded by digestive juices might need to be developed. Perhaps by working with our dental colleagues, patient care and treatment can be improved.

\section{References}

1. Jones RS Jr. The small intestine, section 3 intestinal obstruction. In: Sabiston DC, Lyerly HK, editors. Textbook of surgery: the biological basis of modern 
surgical practice. 15th ed. Philadelphia: WB Saunders, 1997.

2. McFadden DW, Zinner MJ. Manifestations of gastrointestinal disease. In: Schwartz SI, Shiers GT, Spencer FC, editors. Principles of surgery. 6th ed. New York: McGraw-Hill, 1994.

3. Verstocken A, Himpens J, Leman G. An unusual case of small bowel obstruction. Acta Chir Belg 1996;96(4):168-9.

4. Johnson MC, Fisher JK. Plum pit ileus: a case report. Mo Med 1991;88:696-8.

5. Perkins CS. Amalgam appendicitis. Br Dent J 1991; 171:309. 